\title{
Anti-retinal autoantibodies in myopic macular degeneration: a pilot study
}

\author{
Shaun Sebastian Sim $\mathbb{1}^{1,2,3} \cdot$ Chee Wai Wong ${ }^{1,2,3} \cdot$ Quan V. Hoang $\mathbb{1}^{1,2,3,4} \cdot$ Shu Yen Lee ${ }^{1,2,3} \cdot$ Tien Yin Wong ${ }^{1,2,3} \cdot$ \\ Chui Ming Gemmy Cheung ${ }^{1,2,3}$
}

Received: 18 March 2020 / Revised: 15 September 2020 / Accepted: 16 October 2020 / Published online: 28 October 2020

(c) The Author(s), under exclusive licence to The Royal College of Ophthalmologists 2020

\begin{abstract}
Aim The aim of this study is to evaluate the frequency and types of anti-retinal autoantibodies (ARAs) in highly myopic patients and to explore any association between ARAs and the severity of myopic macular degeneration (MMD).

Methods This was a clinic-based study of 16 patients with high myopia (spherical equivalent worse than -6 dioptres or axial length $(\mathrm{AL}) \geq 26.5 \mathrm{~mm}$ ) recruited from the High Myopia clinic of the Singapore National Eye Centre. MMD was graded from fundus photographs according to the Meta-analysis for Pathologic Myopia (META-PM) classification. Severe MMD was defined as META-PM category 3 or 4 . AL and logarithm of the minimal angle of resolution (logMAR) best corrected visual acuity (BCVA) were measured. Sera were obtained from subjects and analysed for the presence of ARAs with the western blot technique.

Results The mean AL was significantly longer in patients with severe MMD $(n=8)$ than those without severe $\operatorname{MMD}(n=8)$ (31.50 vs. 28.51, $p=0.005$ ). There was at least one ARA identified in all patients. The most common ARA was anticarbonic anhydrase II (anti-CAII), present in nine patients (56.3\%). Anti-CAII was detected in more patients with severe MMD than those without ( 75 vs. $37.5 \%, p=0.32)$. LogMar BCVA was also worse in subjects with anti-CAII $(0.5 \pm 0.38$ vs. $0.22 \pm 0.08, p=0.06)$. The number of ARAs significantly correlated with increasing AL $(r=0.61, p=0.012)$.

Conclusions ARAs are prevalent in patients with high myopia, and this increases with increasing AL. In particular, antiCAII antibodies were highly prevalent in patients with severe MMD, suggesting that ARAs may be associated with MMD. Further studies are necessary to confirm these observations in larger cohorts.
\end{abstract}

\section{Introduction}

Pathological myopia is one of the leading causes of irreversible visual loss and is the fourth to ninth most frequent cause of blindness worldwide. The Tajimi Study in Japan showed that myopic macular degeneration (MMD) is the

Chui Ming Gemmy Cheung

gemmy.cheung.c.m@snec.com.sg

1 Singapore National Eye Centre, Singapore, Singapore

2 Singapore Eye Research Institute, Singapore, Singapore

3 Ophthalmology and Visual Sciences Academic Clinical Program, Duke-NUS Medical School, National University of Singapore, Singapore, Singapore

4 Department of Ophthalmology, Edward S. Harkness Eye Institute, Columbia College of Physicians and Surgeons, New York, NY, USA leading cause of legal blindness [1], and it was the second most common cause of visual impairment and blindness in the Beijing Eye Study, China [2]. MMD is characterised by clinical features including lacquer cracks, posterior staphyloma, chorioretinal atrophy and choroidal neovascularization (CNV) [3-5].

There is evidence for the presence of anti-retinal autoantibodies (ARAs) in association with several retinal diseases as such geographic atrophy, age-related macular degeneration (AMD), autoimmune retinopathy and inherited retinal degeneration [6-12]. It has been shown in AMD that the choriocapillaris and the retinal pigment epithelium (RPE) are targets of antibody-mediated complement deposition. The accumulation of immune complexes leads to RPE degeneration and drusen formation. Several studies have showed the association between anti-retinal antibodies and AMD, implicating the immune system in AMD disease progression [13]. Specific autoantibodies to retinal antigens such as anti- $\alpha$-enolase, anti-retinol binding protein 3 
(RBP3), anti-retinol binding protein 1, anti-aldolase $\mathrm{C}$ and anti-pyruvate kinase M2 have been identified in the serum of patients with neovascular AMD and geographic atrophy AMD [9]. In retinitis pigmentosa, it was postulated that autoimmunity developed secondary to damage of blood-retinal barriers, thus exposing retinal antigens to the adaptive immune system [14].

In the highly myopic eye, the blood-retinal barrier can be compromised when the Bruch's membrane is breached, such as in lacquer cracks and myopic CNV. Multimodal imaging studies have revealed the potential breach in the blood-retinal barrier associated with lacquer cracks, which appeared as linear hypocyanescence on indocyanine green angiography and corresponded to areas of partial loss of choriocapillaris on optical coherence tomography (OCT) angiography. It was proposed that disruptions in the choriocapillaris in areas of lacquer cracks occur secondary to mechanical breaks in Bruch's membrane [15]. These and the observation that progressive loss of RPE and choriocapillaris frequently occur around lacquer cracks and sites of previous myopic CNV led to our hypothesis that ARAs could potentially be associated with the chorioretinal atrophy seen in MMD [16, 17]. The aim of this study is to compare the prevalence of ARAs in patients with high myopia with and without severe MMD.

\section{Methods}

\section{Study population}

This was a clinic-based case control study in which patients with high myopia aged 40-80 years, with manifest spherical equivalent (SE) worse than -6.0 dioptres (D) or axial length (AL) of $\geq 26.5 \mathrm{~mm}$ in at least 1 eye, were recruited from the High Myopia clinic of the Singapore National Eye Centre from January 2017 to June 2018. This study was approved by the Centralized Institutional Review Board of SingHealth, Singapore (protocol number R1375/61/2016) and conducted in accordance with the Declaration of Helsinki. All participants provided signed informed consent for their participation. Patients with coexisting or previous ocular disease in either eye known to be associated with ARAs (e.g., AMD, cancer-associated retinopathy, melanoma-associated retinopathy, non-paraneoplastic retinopathy, central serous chorioretinopathy, diabetic retinopathy, retinal dystrophies, macular scarring from any cause other than myopic maculopathy) were excluded. Patients with infectious or non-infectious uveitides, or with systemic autoimmune diseases, malignancies, cognitively impaired or prisoners were also excluded. Cases were defined as subjects with severe myopic maculopathy with either patchy chorioretinal atrophy (Meta-analysis for Pathologic Myopia
(META-PM) category 3) or macular atrophy (META-PM category 4) based on colour fundus photography, and controls were defined as subjects without severe MMD, with tessellated fundus only (META-PM category 1).

\section{Clinical examination}

All patients underwent comprehensive ophthalmic evaluation including best corrected visual acuity (BCVA), measured with the logarithm of the minimal angle of resolution (logMAR) chart (Lighthouse International, New York, New York, USA) at $4 \mathrm{~m}$, slit lamp biomicroscopy examination, and manifest refraction performed by certified study optometrists. SE was calculated as the sum of the spherical power and half of the cylindrical power. The IOL Master (Carl Zeiss Meditec AG, Jena, Germany) was used to measure AL.

\section{Imaging protocol and image grading}

Fundus photography centred at the fovea was performed with a 45-degree digital retinal camera after pupillary dilation with tropicamide $1 \%$ and phenylephrine $2.5 \%$, using Canon CR-DGi with Canon EOS 10D SLR backing (Canon Inc., Tokyo, Japan). The OCT images were obtained using a swept source OCT device (Topcon DRI OCT Triton, Topcon, Tokyo, Japan). This instrument uses a light source with a central wavelength of $1050 \mathrm{~nm}$ and acquisition speed of 100,000 A scans per second. $12 \mathrm{~mm}$ radial scans centred on the macula were obtained. Two retinal specialists (CWW, $\mathrm{DH})$, masked to participant characteristics, performed grading of the fundus photographs using the International META-PM classification system into tessellated fundus, diffuse atrophy, patchy atrophy and macular atrophy [19]. Discrepancies were adjudicated by a senior retinal specialist (CMGC).

\section{Sample analysis}

Patients had their blood samples collected $(\sim 5 \mathrm{ml})$ from the cubital vein, and sent to the Oregon Health \& Science University (OHSU) Ocular Immunology Laboratory for the detection of ARAs (present in cancer-associated retinopathy, melanoma-associated retinopathy and autoimmune retinopathy) via the western blot technique for autoantibodies, such as anti- $\alpha$-enolase, anti-recoverin, etc.

\section{Statistical analysis}

The main outcome measure was the number of ARAs between cases and controls. All data were expressed as mean \pm standard deviation or proportions as appropriate. Means were compared with the independent $t$-test and the 
Fisher's exact test was used to compare the prevalence of ARAs between cases and controls. Correlations between normally distributed continuous variables were analysed with Pearson's correlation coefficient. Multivariate linear regression was performed to analyse associations between ARAs with BCVA, adjusting for age and AL. A $p$ value of $<0.05$ was considered to be statistically significant. All statistical analyses were performed using SPSS version 23 (SPSS, Chicago, Illinois, USA).

\section{Results}

We included a total of 16 subjects. Overall, the mean age was $51.1 \pm 3.0$ years, mean $\mathrm{AL}$ was $30.0 \pm 2.33 \mathrm{~mm}$, mean $\log$ MAR BCVA was $0.38 \pm 0.32$ and 12 patients $(75 \%)$ were female. Table 1 shows the baseline characteristics of eyes with and without severe MMD.

The mean AL was significantly longer in patients with MMD than those without severe MMD (31.50 vs. 28.51, $p=0.005)$. There was no significant difference in age, BCVA and mean number of ARAs between the group with and without severe MMD. There was at least one ARA identified in all patients. The median number of ARAs was 4.5 (range 1-7). The most common ARA was anti-carbonic anhydrase II (anti-CAII, $30 \mathrm{kDa}$ ), which was present in nine patients $(56.3 \%)$, followed by anti-aldolase C (40 kDa) and anti- $\alpha$ enolase $(46 \mathrm{kDa})$, both of which were found in seven patients $(43.8 \%)$.

Anti-CAII autoantibody (30 kDa) was identified in six patients with severe MMD compared to three without severe MMD $(p=0.32)$. Anti-aldolase autoantibody (40 $\mathrm{kDa}$ ) was identified in three patients with severe MMD compared to four without severe MMD $(p=1.0)$. Anti- $\alpha$ enolase autoantibody $(46 \mathrm{kDa})$ was identified in five patients with severe MMD compared to two without severe MMD $(p=0.32)$.

We also analysed the association of three ARAs with the highest frequency and AL and BCVA (Table 2). Patients with anti-CAII autoantibodies had worse vision compared to those without (logMAR BCVA $0.5 \pm 0.38$ vs. $0.22 \pm 0.08, p=0.06$ ), although this result did not reach statistical significance. There was no significant difference in BCVA and AL compared between patients with and without anti-aldolase antibody or anti- $\alpha$ enolase autoantibody (Table 3).

Anti-CAII autoantibodies were significantly correlated with BCVA $(r=0.449, n=16, p=0.04)$. There was no correlation between anti-aldolase $\mathrm{C}$ and anti- $\alpha$ enolase and BCVA. There was also no correlation between anti-CAII, anti-aldolase $\mathrm{C}$ and anti- $\alpha$ enolase and AL. The number of ARAs significantly correlated with increasing AL $(r=0.61$, $p=0.012)$ (Fig. 1).
Linear regression was performed to assess the association between BCVA and anti-CAII, anti-aldolase $\mathrm{C}$ and anti- $\alpha$ enolase autoantibodies. After adjusting for age and AL, we found that BCVA was not significantly associated with any of the three ARAs ( $p=0.17,0.53$ and 0.21 , respectively).

\section{Discussion}

To our knowledge, this is the first clinical study evaluating the association between ARAs and MMD. We found that in our highly myopic patients, all had at least one ARA, with the number of autoantibodies increasing with longer AL. The most common ARA identified was anti-CAII $(30 \mathrm{kDa})$ found in more than half of patients $(56.3 \%$, nine patients) followed by anti-aldolase $\mathrm{C}(40 \mathrm{kDa})$ and anti- $\alpha$ enolase $(46 \mathrm{kDa})$ retinal autoantibodies, both present in $43.8 \%$ of patients. Anti-CAII was detected in higher proportions of highly myopic patients with severe MMD as compared to those without severe MMD (75 vs. $37.5 \% ; p=0.32$ ). The patients with anti-CAII also had worse vision than those without (logMAR BCVA $0.5 \pm 0.38$ vs. $0.22 \pm 0.08, p=$ $0.06)$. Anti-CAII was also significantly correlated with BCVA $(r=0.449, p=0.04)$ but an association between the autoantibody and BCVA was not significant after adjusting for age and $\mathrm{AL}$.

There is increasing evidence in the last two decades describing the association of ARAs in retinopathies, broadly classified into visual paraneoplastic disorders (e.g., CAR or MAR), infection-associated retinopathies (e.g., Onchocerciasis volvulus, Toxoplasmosis gondii, etc.) and retinal degenerative disorders (e.g., RP with cystoid macular oedema, recoverin-associated retinopathy, ARMD, etc.) $[8,10,18-27]$. A prospective study conducted recently identified ARAs in the sera of more than $90 \%$ of retinitis pigmentosa patients with bilateral macular oedema or panretinal degeneration [14]. The authors found that the most common retinal proteins were CAII $(30-\mathrm{kDa})$ and enolase $(46-\mathrm{kDa})$. They postulated that the breakdown in the blood-retinal barrier, a consequence of retinal degenerative process led to the release of retinal proteins that could be antigenic. A previous study by the same group revealed other ARAs with different antigenic specificity altering different metabolic pathways. For example, anti-recoverin antibody was implicated in apoptosis of photoreceptor cells and anti-enolase antibody causing disturbance of retinal cell glycolysis [28, 29]. The presence of ARAs in our highly myopic patients and particularly in those with severe MMD suggests that autoimmunity may be associated with MMD.

We found anti-CAII to be the most common ARA in our study, particularly in patients with severe MMD and was moderately correlated with BCVA. Adamus et al. [30, 31] provided evidence for the pathogenic role of anti-CAII 
Table 1 Baseline characteristics comparing eyes with and without severe MMD.

\begin{tabular}{lccc}
\hline & $\begin{array}{l}{ }^{\mathrm{a}} \text { Patients with severe MMD } \\
(n=8)\end{array}$ & \multicolumn{2}{c}{$\begin{array}{l}{ }^{\mathrm{b}} \text { Patients without severe MMD } \\
(n=8)\end{array}$} \\
\hline Age, years & $51.75 \pm 3.64$ & $50.53 \pm 2.40$ & 0.44 \\
Axial length, mm & $31.50 \pm 1.87$ & $28.51 \pm 1.73$ & 0.005 \\
Best corrected visual acuity, logMAR & $0.39 \pm 0.36$ & $0.36 \pm 0.29$ & 0.85 \\
Mean No. of anti-retinal autoantibodies & $5.0 \pm 1.31$ & $4.0 \pm 1.85$ & 0.24
\end{tabular}

${ }^{a}$ Defined as either patchy chorioretinal atrophy (META-PM category 3) or macular atrophy (META-PM category 4).

${ }^{b}$ Defined as tessellated fundus only (META-PM category 1 ).

Table 2 Comparison of visual acuity and axial length in relation to anti-retinal autoantibody anti-CAII, $30 \mathrm{kDa}$, anti-aldolase $40 \mathrm{kDa}$ and anti- $\alpha$ enolase $(46 \mathrm{kDa})$.

\begin{tabular}{|c|c|c|c|c|c|c|}
\hline \multirow[t]{2}{*}{ Autoantibody } & \multicolumn{3}{|c|}{ Best corrected visual acuity } & \multicolumn{3}{|l|}{ Axial length } \\
\hline & Autoantibody present & Autoantibody absent & $p$ & Autoantibody present & Autoantibody absent & $p$ \\
\hline Anti-CAII (30 kDa) & $0.50 \pm 0.38$ & $0.22 \pm 0.08$ & 0.06 & $30.71 \pm 1.98$ & $29.10 \pm 2.58$ & 0.20 \\
\hline Anti-aldolase C (40 kDa) & $0.45 \pm 0.36$ & $0.32 \pm 0.29$ & 0.44 & $30.40 \pm 1.65$ & $29.70 \pm 2.81$ & 0.54 \\
\hline Anti- $\alpha$ enolase (46 kDa) & $0.28 \pm 0.26$ & $0.45 \pm 0.35$ & 0.30 & $30.42 \pm 2.38$ & $29.68 \pm 2.38$ & 0.55 \\
\hline
\end{tabular}

Table 3 Correlations between number of specific autoantibody with best corrected visual acuity and axial length.

Fig. 1 The correlation between number of anti-retinal autoantibodies with axial length (mm). No of anti-retinal autoantibodies and axial length (mm).

\begin{tabular}{lcccc}
\hline & Best corrected visual acuity & $p$ & Axial length & $p$ \\
\hline Anti-CAII (30 kDa) & 0.449 & 0.04 & 0.353 & 0.09 \\
Anti-aldolase C (40 kDa) & 0.216 & 0.21 & 0.157 & 0.28 \\
Anti- $\alpha$ enolase (46 kDa) & -0.269 & 0.16 & 0.161 & 0.28 \\
\hline
\end{tabular}

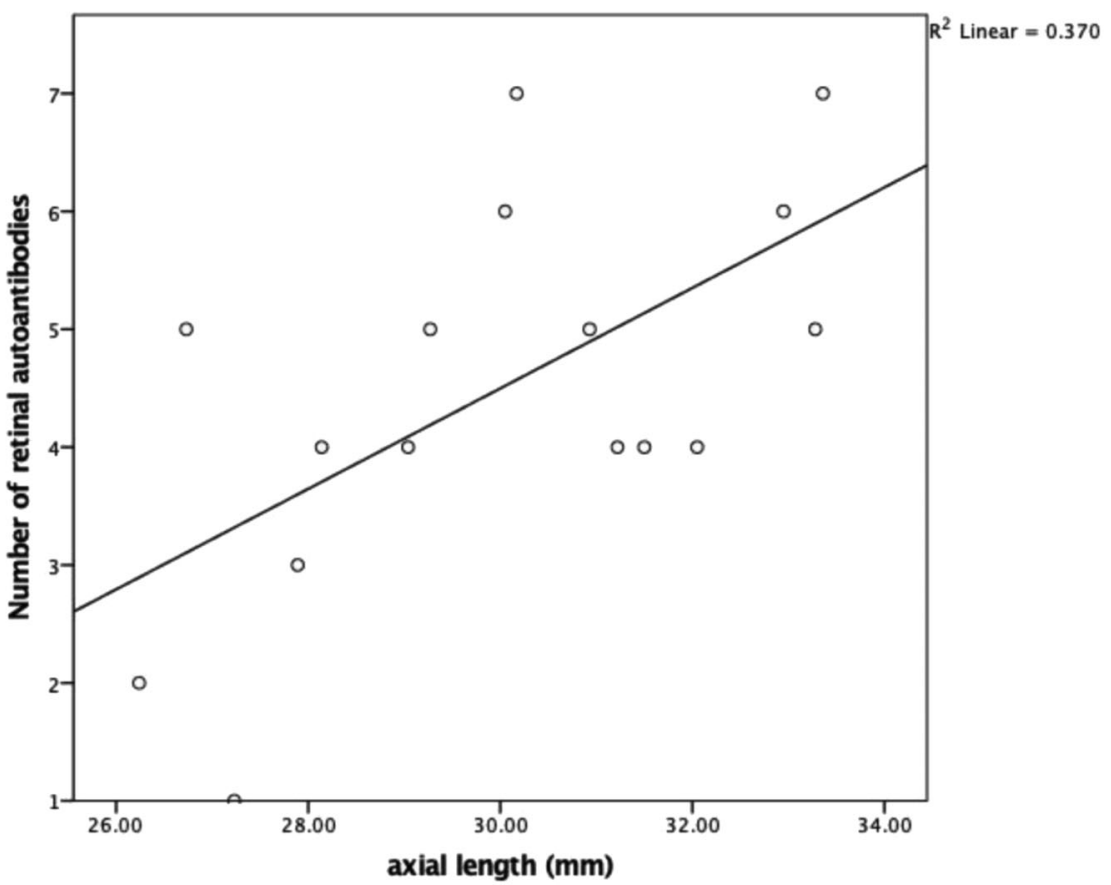


autoantibodies in retinal cell apoptosis. They demonstrated that anti-CAII autoantibodies have the capacity to disrupt metabolic function by inhibiting CAII catalytic activity, decreasing intracellular $\mathrm{pH}$, a cascade that leads to an increase in intracellular calcium and eventually resulting in cell death. We postulate that lacquer cracks, representing breaks in the Bruch's membrane, may expose CAII antigens on RPE cells and lead to immune reactivity with its consequent cascade of the retinal degenerative process.

Other than our proposed mechanism of ARA formation from lacquer cracks, there may be other reasons why ARAs may be seen in high myopes. First, high myopes are at higher risk of retinal detachments. Ichinohasama et al. [32] found significant positive correlation between intravitreal anti-retinal antibodies and macular thickness in a subgroup of eyes with macular off retinal detachment that underwent retinal detachment repair surgery for rhegmatogenous retinal detachment/proliferative vitreoretinopathy. It appears that ARAs formed as a result of the retinal detachment process were related to post-operative cystoid macular oedema, at least in the subgroup of eyes with macular off retinal detachment. None of our highly myopic patients had retinal detachment or retinal surgery that may result in retinal antigen exposure as described above. Second, high myopes are at risk of retinal breaks requiring laser retinopexy, which may predispose to ARA formation from laser induced injury to the retina. To test the hypothesis that retinal injuries may expose retinal antigens to the systemic circulation, Scott et al. [33] performed retinal laser photocoagulation on rabbits, and found circulating ARAs 3 months post treatment. None of our patients had previous retinal laser treatment. Third, white dot syndromes predominantly affect young, highly myopic females. Autoimmune mechanisms have been described related to these conditions, including multiple evanescent white dot syndrome (MEWDS) and punctate inner choroidopathy (PIC) [34-37]. As white dot syndromes typically afflict women more than men [38], our results where all patients had at least one ARA must be taken with the consideration that our series of high myopes had a female predominance $(75 \%)$. None of our patients had typical features for PIC or MEWDS, although it is not possible to be certain that these patients never had these conditions in the past as some patients with MEWDS may have no obvious signs after the acute event.

The limitations of this study include the small sample size which could have influenced the lack of statistical significance of some results. A longitudinal study, with a larger sample size, could better identify the cause and effect relationship between ARAs and the progression of MMD. The inclusion of age-matched, ethnicity-matched controls without myopia would have been ideal. However, for the purpose of this pilot study, we designed the methodology with the focused aim of detecting a difference in the prevalence of autoantibodies in highly myopic patients with severe MMD, and those without severe MMD (META-PM category 1). Further studies, including a non-myopic control group should be performed to validate the findings of this paper. Currently, the presence of ARAs is neither sensitive nor specific for the diagnosis of specific retinopathies. Furthermore, antibody analysis is qualitative and not quantitative; hence its clinical use is fairly limited. There is also a need for standardisation of anti-retinal reactivity for both the development of diagnostic assay systems and for future research studies. Subsequent studies are required to evaluate antibody titres with severe MMD and the role of immune-modulation therapy to alter the progression from mild to severe MMD.

In conclusion, ARAs are prevalent in patients with high myopia, and this increases with increasing AL. In particular, anti-CAII antibodies were highly prevalent in patients with MMD, suggesting that ARAs may be associated with MMD. Further studies are necessary to confirm these observations in larger cohorts.

\section{Summary}

\section{What was known before}

- Association of anti-retinal autoantibodies with retinitis pigmentosa, age-related macular degeneration, etc.

\section{What this study adds}

- The prevalence of anti-retinal autoantibodies in high myopes and specific autoantibodies in association with myopic macular degeneration.

- Correlation of number of retinal autoantibodies with increasing axial length.

Funding This work was supported by an A-STAR Strategic Positioning Fund (SPF2014/002) to SIPRAD, SERI (Singapore Eye Research Institute), IMCB (Institute of Molecular and Cell Biology) Programme in Retinal Angiogenic Diseases and National Medical Research Council Open Fund Large Collaborative Grant: NMRC/ LCG/004/2018 and SNEC HREF-R1375/61/2016.

\section{Compliance with ethical standards}

Conflict of interest The authors declare that they have no conflict of interest.

Publisher's note Springer Nature remains neutral with regard to jurisdictional claims in published maps and institutional affiliations. 


\section{References}

1. Iwase A, Araie M, Tomidokoro A, Yamamoto T, Shimizu H, Kitazawa $\mathrm{Y}$, et al. Prevalence and causes of low vision and blindness in a Japanese adult population: the Tajimi Study. Ophthalmology. 2006;113:1354-62.

2. Xu L, Wang Y, Li Y, Cui T, Li J, Jonas JB. Causes of blindness and visual impairment in urban and rural areas in Beijing: the Beijing Eye Study. Ophthalmology. 2006;113:1134.e1-11.

3. Saw SM, Gazzard G, Shih-Yen EC, Chua WH. Myopia and associated pathological complications. Ophthalmic Physiol Opt. 2005;25:381-91.

4. Silva R. Myopic maculopathy: a review. Ophthalmologica. 2012;228:197-213.

5. Ohno-Matsui K, Kawasaki R, Jonas JB, Cheung CM, Saw SM, Verhoeven VJ, et al. International photographic classification and grading system for myopic maculopathy. Am J Ophthalmol. 2015;159:877-83.e7.

6. Joachim SC, Bruns K, Lackner KJ, Pfeiffer N, Grus FH. Analysis of IgG antibody patterns against retinal antigens and antibodies to alpha-crystallin, GFAP, and alpha-enolase in sera of patients with "wet" age-related macular degeneration. Graefes Arch Clin Exp Ophthalmol. 2007;245:619-26.

7. Forooghian F, Macdonald IM, Heckenlively JR, Héon E, Gordon LK, Hooks JJ, et al. The need for standardization of antiretinal antibody detection and measurement. Am J Ophthalmol. 2008;146:489-95.

8. Milam AH, Saari JC, Jacobson SG, Lubinski WP, Feun LG, Alexander KR. Autoantibodies against retinal bipolar cells in cutaneous melanoma-associated retinopathy. Investig Ophthalmol Vis Sci. 1993;34:91-100.

9. Adamus G, Chew EY, Ferris FL, Klein ML. Prevalence of antiretinal autoantibodies in different stages of age-related macular degeneration. BMC Ophthalmol. 2014;14:154.

10. Adamus G, Ren G, Weleber RG. Autoantibodies against retinal proteins in paraneoplastic and autoimmune retinopathy. BMC Ophthalmol. 2004;4:5.

11. Adamus G. Are anti-retinal autoantibodies a cause or a consequence of retinal degeneration in autoimmune retinopathies? Front Immunol. 2018;9:765.

12. Heckenlively JR. Autoimmune retinopathy patients with antirecoverin immunoreactivity and panretinal degeneration. Arch Ophthalmol. 2000;118:1525.

13. Cherepanoff S, Mitchell P, Wang JJ, Gillies MC. Retinal autoantibody profile in early age-related macular degeneration: preliminary findings from the Blue Mountains Eye Study. Clin Exp Ophthalmol. 2006;34:590-5.

14. Heckenlively JR, Jordan BL, Aptsiauri N. Association of antiretinal antibodies and cystoid macular edema in patients with retinitis pigmentosa. Am J Ophthalmol. 1999;127:565-73.

15. Sayanagi K, Ikuno Y, Uematsu S, Nishida K. Features of the choriocapillaris in myopic maculopathy identified by optical coherence tomography angiography. Br J Ophthalmol. 2017;101: 1524-9.

16. Ohno-Matsui K, Ikuno Y, Lai TYY, Gemmy Cheung CM. Diagnosis and treatment guideline for myopic choroidal neovascularization due to pathologic myopia. Prog Retin Eye Res. 2018;63:92-106.

17. Xu X, Fang Y, Uramoto K, Nagaoka N, Shinohara K, Yokoi T, et al. Clinical features of lacquer cracks in eyes with pathologic myopia. Retina. 2019;39:1265-77.

18. Ohguro H, Ogawa K, Maeda T, Maeda A, Maruyama I. Cancerassociated retinopathy induced by both anti-recoverin and anti- hsc70 antibodies in vivo. Investig Ophthalmol Vis Sci. 1999;40:3160-7.

19. Ohguro H, Ogawa K, Nakagawa T. Recoverin and Hsc 70 are found as autoantigens in patients with cancer-associated retinopathy. Investig Ophthalmol Vis Sci. 1999;40:82-9.

20. Abiose A. Onchocercal eye disease and the impact of Mectizan treatment. Ann Trop Med Parasitol. 1998;92:S11-22.

21. Whittle RM, Wallace GR, Whiston RA, Dumonde DC, Stanford MR. Human antiretinal antibodies in toxoplasma retinochoroiditis. Br J Ophthalmol. 1998;82:1017-21.

22. Weinstein JM, Kelman SE, Bresnick GH, Kornguth SE. Paraneoplastic retinopathy associated with antiretinal bipolar cell antibodies in cutaneous malignant melanoma. Ophthalmology. 1994;101:1236-43.

23. Whitcup SM, Vistica BP, Milam AH, Nussenblatt RB, Gery I. Recoverin-associated retinopathy: a clinically and immunologically distinctive disease. Am J Ophthalmol. 1998;126:230-7.

24. Kim RY, Retsas S, Fitzke FW, Arden GB, Bird AC. Cutaneous melanoma-associated retinopathy. Ophthalmology. 1994;101:1837-43.

25. Sekiguchi I, Suzuki M, Sato I, Ohkawa T, Kawashima H, Tsuchida S. Rare case of small-cell carcinoma arising from the endometrium with paraneoplastic retinopathy. Gynecol Oncol. 1998;71:454-7.

26. Morohoshi K, Goodwin AM, Ohbayashi M, Ono SJ. Autoimmunity in retinal degeneration: autoimmune retinopathy and age-related macular degeneration. J Autoimmun. 2009;33:247-54.

27. Zhu L, Shen W, Zhu M, Coorey NJ, Nguyen AP, Barthelmes D, et al. Anti-retinal antibodies in patients with macular telangiectasia type 2. Investig Ophthalmol Vis Sci. 2013;54:5675-83.

28. Adamus G, Webb S, Shiraga S, Duvoisin RM. Anti-recoverin antibodies induce an increase in intracellular calcium, leading to apoptosis in retinal cells. J Autoimmun. 2006;26:146-53.

29. Magrys A, Anekonda T, Ren G, Adamus G. The role of antialpha-enolase autoantibodies in pathogenicity of autoimmunemediated retinopathy. J Clin Immunol. 2007;27:181-92.

30. Adamus G, Karren L. Autoimmunity against carbonic anhydrase II affects retinal cell functions in autoimmune retinopathy. J Autoimmun. 2009;32:133-9.

31. Adamus G, Machnicki M, Elerding H, Sugden B, Blocker YS, Fox DA. Antibodies to recoverin induce apoptosis of photoreceptor and bipolar cells in vivo. J Autoimmun. 1998;11:523-33.

32. Ichinohasama R, Nishiguchi KM, Fujita K, Aizawa N, Inoue T, Sasaki E, et al. Levels of anti-retinal antibodies in retinal detachment and proliferative vitreoretinopathy. Curr Eye Res. 2018;43:804-9.

33. Scott SE, Bouhenni RA, Chomyk AM, Dunmire JJ, Patil J, Nakamura $\mathrm{H}$, et al. Anti-retinal antibodies in serum of lasertreated rabbits. Investig Ophthalmol Vis Sci. 2012;53:1764-72.

34. Heckenlively JR, Ferreyra HA. Autoimmune retinopathy: a review and summary. Semin Immunopathol. 2008;30:127-34.

35. Jampol LM, Wiredu A. MEWDS, MFC, PIC, AMN, AIBSE, and AZOOR: one disease or many? Retina. 1995;15:373-8.

36. Jacobson SG, Morales DS, Sun XK, Feuer WJ, Cideciyan AV, Gass JD, et al. Pattern of retinal dysfunction in acute zonal occult outer retinopathy. Ophthalmology. 1995;102:1187-98.

37. Neutzner RV, Jäger M, Friedburg C, Deeg CA, Lorenz B. [Blind spot enlargement syndrome in acute zonal occult outer retinopathy with detection of autoantibodies against the retinal antigens CRALBP and S-Ag]. Ophthalmologe. 2011;108:1045-9.

38. Khanna S, Martins A, Oakey Z, Mititelu M. Non-paraneoplastic autoimmune retinopathy: multimodal testing characteristics of 13 cases. J Ophthalmic Inflamm Infect. 2019;9:6. 